Research Journal of Applied Sciences 13 (8): 439-444, 2018

ISSN: $1815-932 \mathrm{X}$

(C) Medwell Journals, 2018

\title{
Algorithm for the Establishment of the Fatigue State for Machine Vision Systems
}

\author{
${ }^{1}$ Robinson Jimenez-Moreno, ${ }^{1}$ Ruben Hernandez and ${ }^{2}$ Javier Martinez-Baquero \\ ${ }^{1}$ Mechatronics Engineering Program, Faculty of Engineering, \\ Militar Nueva Granada University, Bogota, Colombia \\ ${ }^{2}$ Faculty of Basic Science and Engineering, University of Llanos, Villavicencio, Meta-Colombia
}

\begin{abstract}
This study presents the design of an algorithm oriented to the setting of the state of fatigue in a person, based on free software programming tools. It is possible to reproduce the behavior of the state of fatigue and recovery through an open algorithm, that is that can receive information from different sources of acquisition be it visual, electrical or statistical. We obtain a result whose efficiency depends on the source of information for the case it was validated by a video acquisition system using image processing techniques and for which it yields an accuracy in the detection of fatigue of $94.5 \%$.
\end{abstract}

Key words: Fatigue detection, fatigue recovery, microsleep, machine vision, face detection, processing techniques

\section{INTRODUCTION}

The concepts of drowsiness and fatigue are complex phenomena that involve physical, psychosocial and behavioral processes for which different approaches have been used to measure and quantify the aspects that characterize them, since, they compromise the capacity and will to carry out a task (Wijesuriya et al., 2007). Fatigue which decreases the capacity of development in a human, imply the classification of this into two categories, physical and mental fatigue (Grandjean, 1979) where mental fatigue is psychological in nature while physical fatigue is considered synonymous with muscle fatigue.

Mental fatigue is a gradual and cumulative process, associated with reluctance to perform any effort, reduces efficiency and alertness and affects mental performance. Mental fatigue is associated with many factors (Wisner, 1981) such as nutrition, physical health, environment, social relationships and physical activity, among others. The main symptom of mental fatigue is a general feeling of tiredness, feelings of inhibition and reduced activity. There is usually no desire for physical or mental effort and there is an associated feeling of heaviness and drowsiness.

Symptoms of fatigue when exercising a specific task whether of the labor type or not, may present a risk factor for both the person presenting fatigue and those who may be related to it. By Nilsson et al. (1997), it is emphasized that fatigue is an important factor that contributes to the mistakes made by drivers. Thus, the performance of the work of driving versus time requires greater cognitive effort than physical which results in mental fatigue which increases when doing this research for prolonged periods of time by monotonous routes or journeys or by driving after long hours of research. This example is a representative case study in the detection of fatigue, due to the commitment of lives that has charged throughout the world within the most recent developments in this field are found (Zeng et al., 2017; Naz et al., 2017; Chellappa et al., 2016; Jimenez et al., 2014).

Likewise in the conventional industry there is a tendency for the quality control department staff to be responsible for this aspect (Antonio and Fernandez, 2010) research that being routine or prolonged, generates fatigue in the worker, diminishing its performance and compromising the quality of the evaluated product.

These cases, representative of the effect of fatigue on a person are clear evidence of the importance of assessing the physical, environmental and even social conditions that can affect a person over time. At the level of psychology this topic is quite treated and is exposed in several environments as (Dawson et al., 2011; Dorrian et al., 2011; Gonzalez et al., 2011). At the engineering level there are investigations related to the analysis of fatigue in individuals for specific cases such as those presented by Chen et al. (2017) and Al-Libawy et al. (2016) and somewhat more general as discussed by Szypulska and Piotrowski (2012). In these cases they do not focus on developments based on

Corresponding Author: Robinson Jimenez-Moreno, Mechatronics Engineering Program, Faculty of Engineering, Militar Nueva Granada University, Bogota, Colombia 
processing fatigue information under psychological considerations, nor considering the typical recovery effect presented in Helbig Rolf which is addressed in this study. As a complement of techniques of data processing like is exposes by Abdullah (2007).

\section{MATERIALS AND METHODS}

Methods of discrimination of fatigue: Generally, due to the multicausal nature of fatigue, its influence on a person is reflected by the physical, psychological, social, family and personal status. Fatigue can generate short-term effects on the person which are easily detectable but which in the long run could generate major complications such as chronic musculoskeletal conditions, sleep disorders, headaches, etc. A person can notice, due to his individual characteristics, fatigue in a certain time and will suffer its first consequences in a particular way (more muscular, more psychological, more emotional). Likewise, the specific circumstances of the person at that time can influence and modulate the perceived fatigue.

Because fatigue symptoms are usually underestimated mainly in the case of a worker, i.e. when the symptom occurs, the tendency is to recover, according to Boletin Tenico de Ergonomia, fatigue presents an initial phase of "Alarm" that warns of the need for recovery, after which comes a phase of "Resistance". This phase is characterized by an apparent recovery, increased activity and improved performance of the individual. Whoever, experiences it is not usually aware of this situation because he feels active again or full of energy. However, the organism may not respond at any later time, since, it already showed the presence of fatigue. All of the above can lead to psychophysiological factors (Grandjean, 1979), linked to:

- Decreased alertness, mental concentration and motivation

- Reduction of labor capacity

- Weaker and slower muscle contractions

- Muscle tremor and localized pain

- Overload of respiratory, circulatory and neuromuscular functions

- Decreased frequency of Electromyogram (EMG) signal

- Increased accumulation of lactose

- Temperature increase

It is possible, depending on these factors, to set the state of fatigue by means of techniques (Wijesuriya et al., 2007; Grandjean, 1979; Wisner, 1981; Nilsson et al., 1997; Antonio and Fernandez, 2010; Dawson et al., 2011;
Dorrian et al., 2011; Gonzalez et al., 2011; Chen et al., 2017; Al-Libawy et al., 2016; Jimenez-Moreno et al., 2012; Szypulska and Piotrowski, 2012; Yoshitake, 1978; Dinges and Grace, 1998; Caffier et al., 2003; Zeng et al., 2017; Naz et al., 2017; Chellappa et al., 2016; Jimenez et al., 2014; Viola and Jones, 2001; Abdullah, 2007) such as:

Physiological measures evaluating the time required for a subject to show signs of fatigue based on facial expression and/or duration of blinking. Among the visual aspects that can identify fatigue are slow and repetitive head movements, nodding and yawning, parameters that can be validated by analysis using video images. In the same way, it can be validated by measuring the duration of eye closure during blinking, as determined by the Electro Oculography (EOG) signal.

Psychological measures involving a perception of fatigue levels. This type of measurement can be performed using a questionnaire called Chalder Fatigue Scale (CFS), the results of which are derived from the evaluation before and after the monotonous task, so that, the higher scores indicate higher levels of fatigue. Similarly, there are other types of questionnaires applicable to this type of measurement (Yoshitake, 1978).

Measurements regarding propensity for drowsiness measured by means of the Epworth Drowsiness Scale or the Stanford Drowsiness Scale (SSS). Another measure of this style is the assessment of general health through the Lifestyle Assessment Questionnaire (LAQ) which involves a multifactorial assessment of health status, including Body Mass Index (BMI), consumption of alcohol, prescription drugs, cigarette smoking, exercise or additional physical activity, social support, among others.

Electroencephalogram (EEG) and Heart Rate (HR) measurements, including parameters such as Heart Rate Variability (HRV), respiration, peripheral skin temperature and blood pressure. By this method, activity values of the alpha $(8-13 \mathrm{~Hz})$, beta $(14-30 \mathrm{~Hz})$, delta $(0.5-3.5 \mathrm{~Hz})$ and theta $(4-7.5 \mathrm{~Hz})$ waves can be obtained.

Measurements by means of the percentage of closing of the eyes (PERCLOS) (Yoshitake, 1978) which is the average by unit of time in which the eye is closed. Fatigue is related to lack of sleep, pupil diameter, rapid rotation of the eyeball as well as other factors. When defining the average distance and the amount of time needed to close or to fully open the eyes, you can set the speed of opening and closing, such eye closure speed in a sleepy person is clearly different from that of an alert person. By (Dinges and Grace, 1998) the PERCLOS measuring principle is established where times $\mathrm{tl}-\mathrm{t} 4$ are used to measure the closing aperture value, $\mathrm{t} 1$ and $\mathrm{t} 2$ correspond 


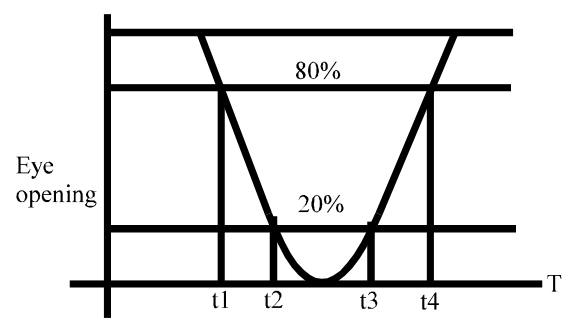

Fig. 1: Percentage of eye closure

to the times in which the eyes are closed from $80 \%$ of opening to $20 \%$ of the opening, $\mathrm{t} 2$ and $\mathrm{t} 4$ to the times of closing to opening between $20 \%$ of opening to $80 \%$ of this:

$$
f=\frac{t_{3}-t_{2}}{t_{4}-t_{1}} \times 100 \%
$$

Figure 1 illustrates the PERCLOS measurement. Figure 1a shows the graphical relationship of the times and percentages in relation to the measurement of the eye, Fig. 1b shows a typical sequence of opening to close of the eye.

Algorithm development: In order to develop an algorithm that allows, as a function of previously acquired data, to replicate the tendency of the fatigue state in a person, aspects such as temporal dependence and the possibility of a recovery state should be considered. By Caffier et al. (2003), the times are associated with the determination of a state of fatigue called microsleep which is characterized by an eye closure in a time span greater than $200 \mathrm{msec}$ while a conventional lashing is in the range of $100 \mathrm{msec}$.

Considering the general behavior trend of fatigue and recovery which is even present in a case such as the microsleep, it is found by Walter that this behavior obeys the one shown in Fig. 2 where non-linear is clear for both fatigue and recovery, this response is not mathematically modeled given the psychological nature of the study presented by Walter and not found in the references consulted.

Since, the fatigue-recovery behavior presents the same tendency, except that one is increasing and the other decreasing, the modeling can be set from either where for the present case it will be derived from the state of recovery.

A decreasing exponential variation is determined by an X-n value, since, there is a system in which the characteristics are to be evaluated in a discrete way, an $n$-weighting sequence will be used. Equation 2 reflects the time behavior of the recovery state according to Fig. 2.
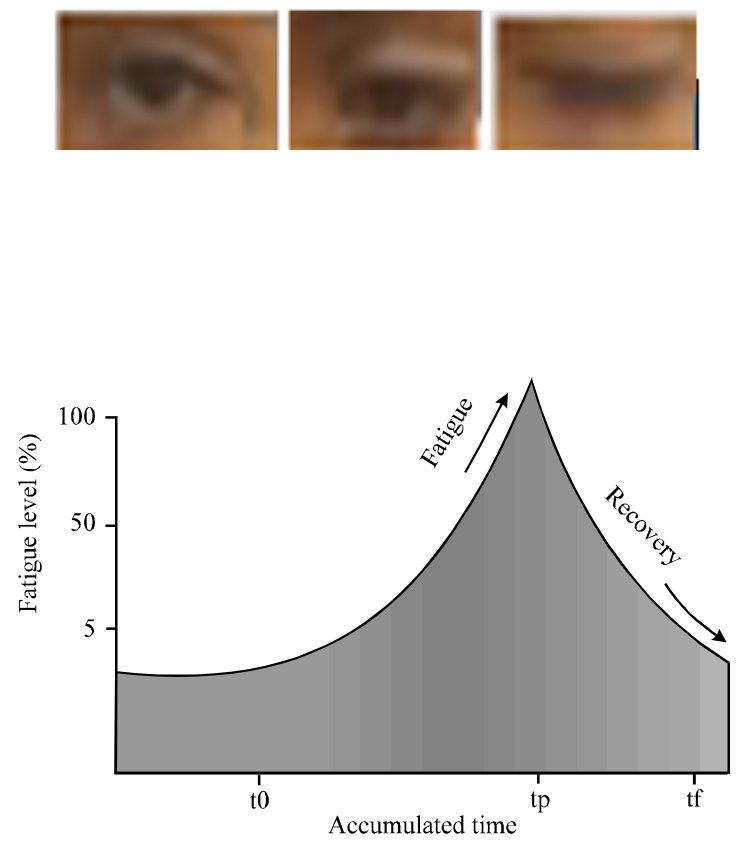

Fig. 2: Tendency of fatigue and recovery. Taken from http://www.ilo.org/safework_bookshelf

$$
\frac{\text { Fatigue }}{\text { Recovery }}=\sum_{\mathrm{i}=0}^{\mathrm{n}} \frac{\left(\alpha+\beta^{*} 2^{-\mathrm{i}}\right)^{*} \mathrm{~F}(\mathrm{t}-\mathrm{i})}{\mathrm{n}+1}
$$

In the equation $\mathrm{n}$ denotes the number of frames with which the fatigue state is to be weighted, $\mathrm{F}(\mathrm{t}-\mathrm{i})$ denotes the current frame that is providing the fatigue state at that time (derived from a data source to be set) and the multiplicative factor is an adjustment, so that, a decrease in the recovery state is generated. This factor must adjust the values of the constants $\alpha$ and $\beta$ to the ratio of the input that is desired to deliver each frame, depending on the number of frames chosen.

The basic information for validation is obtained from an image processing system which evaluates aspects of the person such as state of the eye (opening/closing), nodding and yawning under the algorithms developed by Chen et al. (2017) and Jimenez-Moreno et al. (2012) where the processing time to obtain the $\mathrm{F}(\mathrm{t})$ values are in the order of $40-50 \mathrm{msec}$. Therefore, a final retention factor is generated depending on the last 4 frames, the current and previous 3 frames. This way it can be related the response set between a group of frames and thus, retain the response of the system. This is achieved by the weighted sum of the frames of interest.

For the case with $\mathrm{n}=3$, for validation of four frames, it has $\alpha=0.2$ and $\beta=0.8$ which allows the current validation frame to have the largest contribution. The equation finally developed is shown in Eq. 3: 


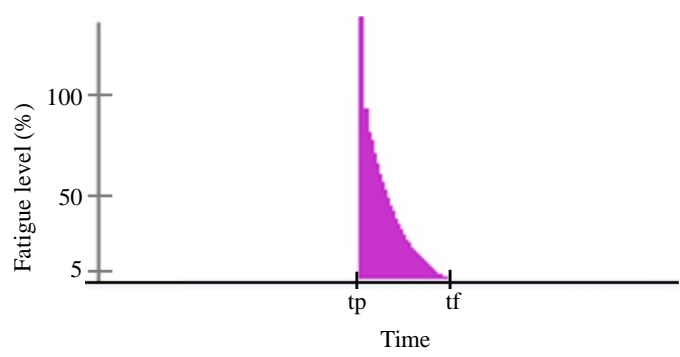

Fig. 3: Response of the algorithm to the state of forgetfulness of fatigue or recovery

$$
\frac{\text { Fat }}{\operatorname{Rec}}=\frac{(0,3 \cdot \mathrm{F}(\mathrm{t}-3)+0,4 \cdot \mathrm{F}(\mathrm{t}-2)+0,6 \cdot \mathrm{F}(\mathrm{t}-1)+\mathrm{F}(\mathrm{t})}{4}
$$

It is emphasized in the multiplicative factor that the fundamental contribution is being made by the current frame, followed by the immediately previous one and in less proportion with almost the same relation the last two, i.e., if the state is not maintained for more than two frames, the fatigue (recovery) begins to be forgotten in contrast if it is maintained, it is reinforced and can go from alert to critical level. The result of this algorithm can be seen in Fig. 3.

\section{RESULTS AND DISCUSSION}

The algorithm was implemented in $\mathrm{C}$ code, under free software tools, using the openCV image processing library. The computer has a Dual Core $1.8 \mathrm{GHz}$ processor and a 4 GB RAM. For tests of the algorithm it was set an extraction of facial characteristics by means of image processing that allowed to infer by the development the state of fatigue of the person in front of the camera.

The image processing algorithm that identifies the face and eyes within the captured image is based on the Viola-Jones algorithm that operates on the basis of a trained AdaBoost classifier (Viola and Jones, 2001). This algorithm requires a large number of images $\left(\mathrm{x}_{\mathrm{n}}\right)$ to train the classifier, including images of faces called positive images $\left(y_{n}=1\right)$ and likewise of faceless images or negative images $\left(\mathrm{y}_{\mathrm{n}}=0\right)$, conforming the arrangement described in Eq. 4:

$$
\mathrm{X}_{[\mathrm{n}]}=\left[\left(\mathrm{x}_{1}, \mathrm{y}_{1}\right),\left(\mathrm{x}_{2}, \mathrm{y}_{2}\right), \ldots,\left(\mathrm{x}_{\mathrm{n}}, \mathrm{y}_{\mathrm{n}}\right)\right]
$$

Depending on these images their characteristics are extracted using Haar descriptors, such as those illustrated in Fig. 4. Each characteristic is a single value obtained by subtracting the sum of the pixels of the image that cover the area of the white rectangle from the sum of the pixels under the black rectangle which forms an integral image in

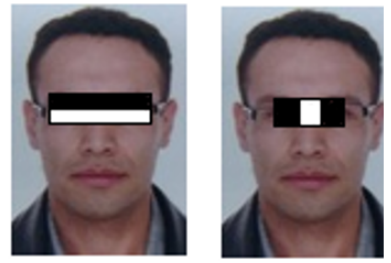

Fig. 4: Haar descriptors

relation to the learning relations of each characteristic area to learn. For example, the area of the eyes on the face as seen on the reticular part of Fig. 4.

AdaBoost constructs a final classification rule using several classifiers which because of their simplicity and low precision are called "Weak" classifiers which when used together allow a more precise classifier, called "Strong".

The characteristics obtained are evaluated by a weak classifier which decides if the sub-image of the search window used corresponds to a face or not. The final classifier $(\mathrm{h}(\mathrm{x}))$ is a weighted sum of weak classifiers. To determine this, it is selected the characteristics with a minimum error rate $(\epsilon, j)$, initially giving the same weight to each image, increasing the value of each weight $(w)$ to the misclassified images and calculating a new error rate, through an iterative process. The process continues until the required precision is reached, under the following algorithm 1:

\section{Algorithm 1; Precision:}

Step 1

for $\mathrm{i}=1$ to $\mathrm{n}$

$\mathrm{X}[\mathrm{n}]=\left[\left(\mathrm{x}_{1}, \mathrm{y}_{1}\right) \ldots . .\left(\mathrm{x}_{\mathrm{n}}, \mathrm{y}_{\mathrm{n}}\right)\right]$

wi $=1 /(2 \mathrm{~m}), 1 /(2 \mathrm{l})$

Step 2

for $\mathrm{t}=1$ to $\mathrm{T}$

$$
\begin{aligned}
& W_{t, i} \leftarrow \frac{W_{t, i}}{\sum_{j=1}^{n} W_{t, j}} \\
& \epsilon_{j}=\sum_{i} W_{i\left|h_{j}\left(x_{1}\right)-y_{1}\right|} \\
& W_{t+1, i}=W_{t, i \beta_{t}}^{1-\epsilon_{1}}
\end{aligned}
$$

Step 3

$$
\begin{gathered}
h(x)= \begin{cases}1 s i \sum_{t=1}^{T} \alpha_{t} h_{t}(x) \geq \frac{1}{2} \sum_{t=1}^{T} \alpha_{t} \\
0 & \text { Othercase }\end{cases} \\
\alpha_{t}=\frac{\log 1}{\beta_{t}}
\end{gathered}
$$

With

The characteristics to analyze, extracted from the discrimination of the face and the eyes, corresponds to 


\begin{tabular}{lll}
\multicolumn{2}{l}{ Table 1: Confusion matrix of fatigue detection } \\
& Predicted & \\
& --- Positive & \\
Fatigue detection & & 17860 \\
Real & 31 & 51 \\
False & 534 & \\
True & 99.5 & \\
Accuracy (\%) & 94.51 & \\
Precision (\%) &
\end{tabular}

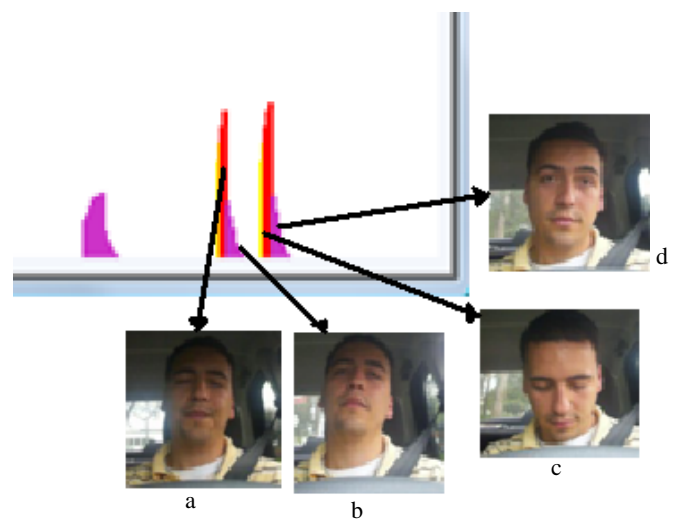

Fig. 5: Response of the algorithm to a fatigue input

the analysis of nodding and closing of eyes by PERCLOS, as it is developed in a previous research (Jimenez-Moreno et al., 2012).

A test video is used in which a series of fatigue conditions were simulated, including yawning, nodding and sleep or sleepiness episodes when performing a daily activity such as driving. Figure 5 shows the algorithm's response in which two episodes of microsleep (a and c) are presented in which the second (c) includes nodding. Following each of these is a state of recovery or sudden awakening ( $b$ and $d)$.

Table 1 illustrates the result obtained by the algorithm when validating in the whole video sequence, composed in this case by 18476 frames or image frames by means of a confusion matrix which relates cases manually labeled in each frame as fatigue state which are considered true positive (VP). The other cases listed in the table correspond in the case of False Positives (FP) to the states of no fatigue or recovery that the algorithm identifies as fatigue, the False Negatives (FN) correspond to those cases of non-fatigue that the algorithm correctly identified which is why the high number of frames presented (17860) (Fig. 5).

The system accuracy is calculated by means of Eq. 5 which shows the high degree obtained, allowing to infer that the system operates satisfactorily:

$$
\text { Accuracy }=\frac{\mathrm{VP}+\mathrm{FN}}{\mathrm{VP}+\mathrm{FN}+\mathrm{VN}+\mathrm{FP}}
$$

\section{CONCLUSION}

It was possible to implement a replication algorithm of the behavior of fatigue in a person which when applied to a data acquisition system, adequately described psychological studies of such behavior.

By validating the algorithm's functionality by means of a video sequence simulating fatigue episode, a performance in accuracy of the $94.51 \%$ was found by means of a confusion matrix analysis where the failure rate is determined by the inherent failures of the machine vision algorithm and can be replicated to a greater or lesser degree if other sources of information acquisition are used.

\section{ACKNOWLEDGEMENT}

The research for this study was supported by Nueva Granada Military University and University of Llanos.

\section{REFERENCES}

Abdullah, S., 2007. The wavelet transform for fatigue history editing: Is it applicable for automotive applications. J. Eng. Applied Sci., 2: 342-349.

Al-Libawy, H., A. Al-Ataby, W. Al-Nuaimy, M.A. Al-Taee and Q. Al-Jubouri, 2016. Fatigue detection method based on smartphone text entry performance metrics. Proceedings of the 2016 9th International Conference on Developments in eSystems Engineering (DeSE), August 31-September 2, 2016, IEEE, Liverpool, UK., ISBN:978-1-50905488-6, pp: 40-44.

Antonio, J. and D.V.P. Fernandez, 2010. Process Management. 4th Edn., ESIC, France, ISBN:978-84-7356-697-1, Pages: 335.

Caffier, P.P., U. Erdmann and P. Ullsperger, 2003. Experimental evaluation of eye-blink parameters as a drowsiness measure. Eur. J. Appl. Physiol., 89: 319-325.

Chellappa, Y., N.N. Joshi and V. Bharadwaj, 2016. Driver fatigue detection system. Proceedings of the 2016 IEEE International Conference on Signal and Image Processing (ICSIP), August 13-15, 2016, IEEE, Beijing, China, ISBN: 978-1-5090-2378-3, pp: 655-660.

Chen, J., Y. Tao, D. Zhang, X. Liu and Z. Fang et al., 2017. Fatigue detection based on facial images processed by difference algorithm. Proceedings of the 13th IASTED International Conference on Biomedical Engineering (BioMed), February 20-21, 2017, IEEE, Innsbruck, Austria, ISBN:978-1-5090-4908-0, pp: 208-211. 
Dawson, D., Y.I. Noy, M. Harma, T. Akerstedt and G. Belenky, 2011. Modelling fatigue and the use of fatigue models in work settings. Accid. Anal. Prev., 43: $549-564$.

Dinges, D.F. and R. Grace, 1998. PERCLOS: A valid psychophysiological measure of alertness as assessed by psychomotor vigilance. Federal Highway Administration, Washington, DC., USA.

Dorrian, J., S.D. Baulk and D. Dawson, 2011. Work hours, workload, sleep and fatigue in Australian rail industry employees. Appl. Ergon., 42: 202-209.

Gonzalez, C., B. Best, A.F. Healy, J.A. Kole and L.E. Bourne, 2011. A cognitive modeling account of simultaneous learning and fatigue effects. Cognit. Syst. Res., 12: 19-32.

Grandjean, E., 1979. Fatigue in industry. Occup. Environ. Med., 36: 175-186.

Jimenez, M.R., O.A. Sanchez and D.A. Hurtado, 2014. Driver distraction detection using machine vision techniques. Eng. Competitiveness, 16: 55-63.

Jimenez-Moreno, R., S.A. Orjuela-Vargas, P. Van-Hese, F. Prieto and V. Grisales et al., 2012. Video surveillance for monitoring driver's fatigue and distraction. Proceedings of the Conference on Optics, Photonics and Digital Technologies for Multimedia Applications II Vol. 8436, April 17-18, 2012, SPIE, ýBellingham, Washington, USA., ISBN: 9780819491282, pp: 25-32.

Naz, S., A. Ahmed, Q. Ain-ul-Mubarak and I. Noshin, 2017. Intelligent driver safety system using fatigue detection. Proceedings of the 2017 19th International Conference on Advanced Communication Technology (ICACT), February 19-22, 2017, IEEE, Bongpyeong, South Korea, ISBN:978-1-5090-4892-2, pp: 89-93.
Nilsson, T., T.M. Nelson and D. Carlson, 1997. Development of fatigue symptoms during simulated driving. Accid. Anal. Prev., 29: 479-488.

Szypulska, M. and Z. Piotrowski, 2012. Prediction of fatigue and sleep onset using HRV analysis. Proceedings of the 19th International Conference on Mixed Design of Integrated Circuits and Systems (MIXDES), May 24-26, 2012, IEEE, Warsaw, Poland, ISBN: :978-1-4577-2092-5, pp: 543-546.

Viola, P. and M. Jones, 2001. Rapid object detection using a boosted cascade of simple features. Proceedings of the 2001 IEEE Computer Society Conference on Computer Vision and Pattern Recognition(CVPR 2001) Vol. 1, December 8-14, 2001, IEEE, Kauai, Hawaii, USA., ISBN:0-7695-1272-0, pp: I-511-I-518.

Wijesuriya, N., Y. Tran and A. Craig, 2007. The psychophysiological determinants of fatigue. Int. J. Psychophysiol., 63: 77-86.

Wisner, A., 1981. Organizational Stress, Cognitive Load and Mental Suffering. In: Machine Pacing and Occupational Stress, Salvendy, G. and M. Smith (Eds.). Taylor and Francis, London, England, UK., pp: $37-44$.

Yoshitake, H., 1978. Three characteristic patterns of subjective fatigue symptoms. Ergon., 21: 231-233.

Zeng, S., J. Li, L. Jiang and J. Jiang, 2017. A driving assistant safety method based on human eye fatigue detection. Proceedings of the 29th Chinese Conference on Control and Decision (CCDC), May 28-30, 2017, IEEE, Chongqing, China, ISBN: 978-1-5090-4658-4, pp: 6370-6377. 